\title{
Effective Conversion of Metmyoglobin to Oxymyoglobin by Cysteine-Substituted Polyphenols
}

Sari Honda, Yukari Miura, Toshiya Masuda, Akiko Masuda

\begin{tabular}{|c|l|}
\hline Citation & Journal of Agricultural and Food Chemistry, 64(4); 806-811 \\
\hline Issue Date & $2016-01-12$ \\
\hline Type & Journal Article \\
\hline Textversion & Author \\
\hline Rights & $\begin{array}{l}\text { This document is the Accepted Manuscript version of a Published Work that appeared } \\
\text { in final form in Journal of Agricultural and Food Chemistry, copyright } @ \text { American } \\
\text { Chemical Society after peer review and technical editing by the publisher. To access } \\
\text { the final edited and published work see https://doi.org/10.1021/acs.jafc.5b05511. }\end{array}$ \\
\hline DOI & \begin{tabular}{l} 
10.1021/acs.jafc.5b05511 \\
\hline
\end{tabular} \\
\hline
\end{tabular}

\author{
Self-Archiving by Author(s) \\ Placed on: Osaka City University
}

HONDA, S., MIURA, Y., MASUDA, T., \& MASUDA, A. (2016). Effective Conversion of Metmyoglobin to Oxymyoglobin by Cysteine-Substituted Polyphenols. Journal of Agricultural and Food Chemistry. 64, 806-811. https://doi.org/10.1021/acs.jafc.5b05511 


\title{
Effective Conversion of Metmyoglobin to Oxymyoglobin by Cysteine-Substituted Polyphenols
}

\author{
Sari Honda, ${ }^{\dagger, \dagger}$ Yukari Miura, ${ }^{\ddagger}$ Toshiya Masuda, ${ }^{\dagger, *}$ and Akiko Masuda ${ }^{\S}$ \\ ${ }^{\dagger}$ Graduate School of Human Life Science, Osaka City University, Osaka 558-8585, \\ Japan \\ ${ }^{\star}$ Graduate School of Integrated Arts and Science, Tokushima University, Tokushima \\ 770-8502, Japan

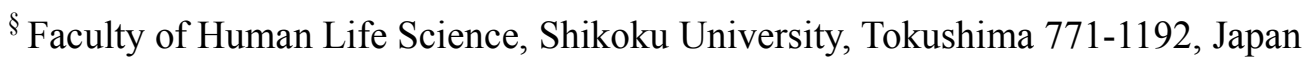

Running Head: Reducing-effects of cysteinyl polyphenols on metmyoglobin

*Corresponding Author

(FAX: +81-6-6605-2813; E-mail: masuda_t@life.osaka-cu.ac.jp) 
ABSTRACT: Reaction products from the peroxidase-catalyzed oxidation of polyphenols in the presence of cysteine showed a potent activity for reducing metmyogolobin (MetMb) to bright-colored oxymyogolobin $\left(\mathrm{MbO}_{2}\right)$. High-performance liquid chromatography (HPLC) purification of the reaction products from catechin, chlorogenic acid, dihydrocaffeic acid, hydroxytyrosol, nordihydroguaiaretic acid, and rosmarinic acid afforded corresponding $S$-cysteinyl compounds, whose structures were determined by nuclear magnetic resonance (NMR) spectroscopy and mass spectrometry (MS). The isolated cysteinyl polyphenols showed a concentration-dependent reducing activity for MetMb to $\mathrm{MbO}_{2}$ for initial one hour. However, after one hour, some of them decreased the amount of $\mathrm{MbO}_{2}$ produced. The effect of the number of cysteinyl sulfur substitutions in polyphenols on both MetMb reduction and $\mathrm{MbO}_{2}$ maintenance was examined using hydroxytyrosols with different numbers of cysteine substitutions; these hydroxytyrosols were synthesized from hydroxytyrosol and an $N$-acetylcysteine methyl ester. The hydroxytyrosol derivative that substituted with two $\mathrm{N}$-acetylcysteine esters exhibited the most effective reducing activity without any effect on $\mathrm{MbO}_{2}$.

KEYWORDS: metmyoglobin reducing activity, cysteinyl polyphenols, peroxidase-catalyzed oxidation, bright meat color preservation 


\section{INTRODUCTION}

The bright red color of meat is typically an indication of its freshness. The red color is the result of oxymyoglobin $\left(\mathrm{MbO}_{2}\right)$, which is formed during the blooming process of meat when molecular oxygen reacts with the heme protein, myoglobin. It is well known that myoglobin exists in various redox states, depending on the state of the chelated Fe ion. Myoglobin is rapidly converted to $\mathrm{MbO}_{2}$ in an oxygen atmosphere; however, the produced $\mathrm{MbO}_{2}$ is not very stable and is readily oxidized to a brown-colored metmyoglobin (MetMb). This browning reduces the market value of fresh meat. ${ }^{1}$

Therefore, the preservation of the bright red color of meat and meat products is very important for the food industry. ${ }^{2}$

Polyphenols are well-known bioactive constituents of plants and plant-derived foods. They have potent reducing properties, resulting in the inhibition of oxidative deterioration of food. We have previously attempted to utilize such polyphenols to prevent the oxidative browning of $\mathrm{MbO}_{2}$. However, the potent antioxidant polyphenols actually accelerated the oxidation of $\mathrm{MbO}_{2}$ to MetMb. ${ }^{3}$ This effect could be attributed the pro-oxidant properties of these highly active polyphenols. As an alternative approach to obtain fresh red-colored meat, we attempted to reduce MetMb back to $\mathrm{MbO}_{2}{ }^{4}$ The results showed that flavonol polyphenols such as quercetin and kaempferol 
could partially convert MetMb back to $\mathrm{MbO}_{2}$, and reduced production of the pro-oxidant quinone derivatives was identified as a possible mechanism. However, other polyphenols previously reported with a strong reducing potency could not produce sufficient amounts of $\mathrm{MbO}_{2}$. We found that the addition of equimolar amounts of cysteine to the polyphenol solution was very effective. ${ }^{5}$ A main reason for this was that the pro-oxidant quinones, which were formed in the assay system, were quenched by the nucleophilic addition of the cysteinyl thiol. In another investigation, we also found that several cysteine-substituted polyphenols could enhance the antioxidant capacity against lipid oxidation. ${ }^{6}$ These results indicated that such cysteine-substituted polyphenols exhibited a useful enhancing property for the MetMb reducing and the maintenance of $\mathrm{MbO}_{2}$, which is very important for preserving the color of fresh meat. In this investigation, we prepared various cysteine-substituted polyphenols and examined their MetMb reducing activity. We also investigated the effect of the number of $S$-cysteinyl-substitutions on the ability of the polyphenols to reduce MetMb using hydroxytyrosol.

\section{MATERIALS AND METHODS}

Chemicals and instruments. Myoglobin, gentisic acid (purity >98\%), 
protocatechuic acid (purity $>97 \%$ ), quercetin dihydrate (purity $>95 \%$ ), potassium hexacyanoferrate (III), sodium hydrosulfite, cysteine, and $N$-butoxycarbonylcysteine were obtained from Nacalai Tesque (Kyoto, Japan). $N$-Acetyl cysteine (purity $>98 \%$ ), caffeic acid (purity $>98 \%$ ), hydroxytyrosol (purity $>98 \%$ ), myricetin (purity $>97 \%$ ), morin (purity $>90 \%$ as hydrate), and kaempferol (purity $>97 \%$ as hydrate) were purchased from Tokyo Kasei (Tokyo, Japan). Catechin (purity $>98 \%$ as hydrate), rosmarinic acid (purity $>97 \%$ ), chlorogenic acid (purity $>95 \%$ ), nordihydroguaiaretic acid (purity $>97 \%$ ), and dihydrocaffeic acid (purity $>98 \%$ ) were obtained from Sigma-Aldrich (St. Louis, MO). Taxifolin (purity $>96 \%$ ) was obtained from Funakoshi (Tokyo, Japan). Peroxidase (from horseradish) was purchased from Toyobo (Osaka, Japan). All organic solvents (extra pure grade or HPLC grade) were obtained from Nacalai Tesque. Distilled water produced by a water-distilling instrument, SA-2100E (EYELA, Tokyo, Japan), was used for all experiments. NMR spectra were obtained from an ECS-400 spectrometer (JEOL, Tokyo, Japan) or an AVANCE 300N spectrometer (Bruker BioSpin, Yokohama, Japan) using the manufacturer's pulse sequences for ${ }^{1} \mathrm{H},{ }^{13} \mathrm{C}$, HH-COSY, NOESY, HMQC, and HMBC measurements. High-resolution mass spectrometric data were obtained using a XEVO QtofMS spectrometer (Waters Japan, Tokyo, Japan) in ESI mode and determination of each 
molecular formula from the MS data was carried out using MassLynx v.4.1 software (Waters Japan). An LC-20AT low-pressure gradient system (Shimadzu, Kyoto, Japan) equipped with an SPD-M20A photodiode array detector (PDA) was employed for analytical HPLC. PDA data were analyzed using LabSolutions ver. 5.51 (Shimadzu). An LC-6AD system (Shimadzu) equipped with a UV-8011 detector (Tosoh, Tokyo, Japan) was used for preparative HPLC.

Preparation of MetMb. The MetMb solution was prepared from a stock solution of $\mathrm{MbO}_{2}$, which was prepared using a method reported previously. ${ }^{4}$ Potassium hexacyanoferrate (III) $(2 \mathrm{mg})$ was added to a solution of $\mathrm{MbO}_{2}(120 \mu \mathrm{mol} / \mathrm{L})$ in a phosphate buffer $(50 \mathrm{mmol} / \mathrm{L}, \mathrm{pH} 7.4,3 \mathrm{~mL})$ at $23{ }^{\circ} \mathrm{C}$. After gentle stirring for $0.5 \mathrm{~min}$, the solution was desalted three times at $14,000 \mathrm{~g}$ for $6 \mathrm{~min}$, at $4{ }^{\circ} \mathrm{C}$, with an Amicon Ultra-0.5, Ultracel-10 ultrafiltration membrane (Merck Millipore, Cork, Ireland) using phosphate buffer. The final residual solution $(\approx 0.5 \mathrm{~mL})$ was diluted with buffer, yielding $120 \mu \mathrm{mol} / \mathrm{L}$ of the MetMb solution $(3 \mathrm{~mL})$. The purity and concentration of the MetMb solution were confirmed using a UVmini 1240 UV/VIS spectrometer (Shimadzu). ${ }^{7,8}$

Coupling Reaction between Polyphenols and Cysteine. To a straight vial of the solution of each polyphenol in methanol $(18 \mathrm{mmol} / \mathrm{L}, 1 \mathrm{~mL})$, cysteine in water $(18$ 
$\mathrm{mmol} / \mathrm{L}, 1 \mathrm{~mL})$, peroxidase in water $(60 \mu \mathrm{mol} / \mathrm{L}, 20 \mu \mathrm{L})$, and $\mathrm{H}_{2} \mathrm{O}_{2}(400 \mathrm{mmol} / \mathrm{L}, 30$ $\mu \mathrm{L}$ ) were subsequently added and then the solution was made up to $4 \mathrm{~mL}$ with water. The solution was incubated at $25^{\circ} \mathrm{C}$ for $8 \mathrm{~min}$ to afford oxidized polyphenol solution (4.5 mmol/L based on starting polyphenol concentration). Control experiments were carried out by similar procedure without addition of cysteine to make reference samples.

Measurement of MetMb reduction to $\mathrm{MbO}_{2}$. Briefly, $50 \mathrm{mmol} / \mathrm{L}$ of phosphate buffer ( $\mathrm{pH} 7.4,250 \mu \mathrm{L}), 120 \mu \mathrm{mol} / \mathrm{L}$ of MetMb $(150 \mu \mathrm{L}$; in the same buffer), DMSO $10 \mu \mathrm{L}$, and the sample solution mentioned above $(4.5 \mathrm{mmol} / \mathrm{L}, 40 \mu \mathrm{L})$ were subsequently added to a 96-well microplate. The microplate was then incubated at $37^{\circ} \mathrm{C}$ in a Multiskan GO microplate reader (Thermo Fisher Scientific, Yokohama, Japan) for 1 $\mathrm{h}$, and absorbance was measured at 500 and $582 \mathrm{~nm}$. The $\mathrm{MbO}_{2}$ and MetMb concentrations in the wells were calculated using the following equation ${ }^{5}: \mathrm{MbO}_{2}$ $(\mu \mathrm{mol} / \mathrm{L})=\left(89.7 \times \mathrm{A}_{582}\right)-\left(32.9 \times \mathrm{A}_{500}\right)$ where $\mathrm{A}_{500}$ and $\mathrm{A}_{582}$ refer to absorbance at 500 $\mathrm{nm}$ and $582 \mathrm{~nm}$, respectively. The reductive conversion efficiency from MetMb to $\mathrm{MbO}_{2}$ was expressed as a percentage by calculating the $\mathrm{MbO}_{2}$ concentration relative to the initial concentration $(60 \mu \mathrm{mol} / \mathrm{L})$ of MetMb.

\section{Purification and Structure Identification of Cysteinyl Polyphenols.}

Preparative HPLC conditions are summarized in a table in supporting information 
section. HR-MS and NMR data of the following synthesized compounds are also summarized in the section.

Cysteinyl catechins. To a catechin $(50 \mathrm{mg})$ solution in methanol $(4 \mathrm{~mL})$ and water (12 $\mathrm{mL}$ ) were added cysteine $(42 \mathrm{mg})$, peroxidase $(4.6 \mathrm{mg})$, and $\mathrm{H}_{2} \mathrm{O}_{2}(0.4 \mathrm{~mol} / \mathrm{L}, 360 \mu \mathrm{L})$ with stirring at $25^{\circ} \mathrm{C}$. After several minutes, methanol in the solution was removed in vacuo and the resulting solution was purified by preparative HPLC to give 2 '-S-cysteinylcatechin (1) and 5'-S-cysteinylcatechin (2) in 16 and 22\% yields, respectively.

Cysteinyl chlorogenic acid. A chlorogenic acid $(50 \mathrm{mg})$ solution in methanol (3 mL) and water $(10 \mathrm{~mL})$, cysteine $(103 \mathrm{mg})$, peroxidase $(3.7 \mathrm{mg})$, and $\mathrm{H}_{2} \mathrm{O}_{2}(0.4 \mathrm{~mol} / \mathrm{L}, 350$ $\mu \mathrm{L}$ ) were treated under similar conditions to those of catechin and the obtained reaction mixture was purified by preparative HPLC to give 2 "-S-cysteinylchlorogenic acid (3) in $64 \%$ yield.

Cysteinyl dihydrocaffeic acids: A dihydrocaffeic acid (50 mg) solution in methanol (7 $\mathrm{mL})$ and water $(18 \mathrm{~mL})$, cysteine $(131 \mathrm{mg})$, peroxidase $(6.6 \mathrm{mg})$, and $\mathrm{H}_{2} \mathrm{O}_{2}(0.4 \mathrm{~mol} / \mathrm{L}$, $680 \mu \mathrm{L}$ ) were treated under similar conditions to those of catechin and the obtained reaction mixture was purified by preparative HPLC to give 2',5'-bis( $S$-cysteinyl)dihydrocaffeic acid (4) and 5'-S -cysteinyldihydrocaffeic acid (5) in 
$8 \%$ and $65 \%$ yields, respectively.

Cysteinyl hydroxytyrosols: A hydroxytyrosol $(50 \mathrm{mg})$ solution in methanol $(5 \mathrm{~mL})$ and water $(10 \mathrm{~mL})$, cysteine $(78 \mathrm{mg})$, peroxidase $(8.1 \mathrm{mg})$, and $\mathrm{H}_{2} \mathrm{O}_{2}(0.4 \mathrm{~mol} / \mathrm{L}, 350 \mu \mathrm{L})$ were treated under similar conditions to those of catechin and the obtained reaction mixture was purified by preparative HPLC to give $2^{\prime}, 5^{\prime}$-bis( $S$-cysteinyl)hydroxytyrosol (6) and 5'-S-cysteinylhydroxytyrosol (7) in 5\% and $46 \%$ yields, respectively.

Cysteinyl nordihydroguaiaretic acid: A nordihydroguaiaretic acid (50 mg) solution in methanol $(6 \mathrm{~mL})$ and water $(10 \mathrm{~mL})$, cysteine $(40 \mathrm{mg})$, peroxidase $(4.4 \mathrm{mg})$, and $\mathrm{H}_{2} \mathrm{O}_{2}$ $(0.4 \mathrm{~mol} / \mathrm{L}, 450 \mu \mathrm{L})$ were treated under similar conditions to those of catechin and the obtained reaction mixture was purified by preparative HPLC to give 5 '-S-cysteinylnordihydroguaiaretic acid (8) in $20 \%$ yield.

Cysteinyl rosmarinic acid: A rosmarinic acid $(50 \mathrm{mg})$ solution in methanol $(3 \mathrm{~mL})$ and water $(10 \mathrm{~mL})$, cysteine $(101 \mathrm{mg})$, peroxidase $(3.5 \mathrm{mg})$, and $\mathrm{H}_{2} \mathrm{O}_{2}(0.4 \mathrm{~mol} / \mathrm{L}, 350 \mu \mathrm{L})$ were treated under similar conditions to those of catechin and the obtained reaction mixture was purified by preparative HPLC to give $2^{\prime \prime \prime}-S$-cysteinylrosmarinic acid (9) in $48 \%$ yield.

Preparation of 5'-S-(N-acetyl-O-methylcysteinyl)hydroxytyrosol (10) and $2^{\prime}, 5^{\prime}\left(\right.$ or $\left.5^{\prime}, 6^{\prime}\right)-S$-bis $[(N$-acetyl-O-methyl)cysteinyl $]$ hydroxytyrosol (11). To a 
solution of hydroxytyrosol (400 mg) and $N$-acetylcysteine methyl ester $(551 \mathrm{mg})$ in acetonitrile $(250 \mathrm{~mL})$ was added slowly a fine powder of 2,2-diphenyl-1-picrylhydrazyl $(\mathrm{DPPH}, 2.1 \mathrm{~g})$ with stirring at $23{ }^{\circ} \mathrm{C}$. The solution was then evaporated in vacuo and filtered after addition of a small amount of the mixed solvent from ethyl acetate and acetone $[6: 1(\mathrm{v} / \mathrm{v})]$. The filtrate was subjected to silica gel column chromatography eluted with ethyl acetate-acetone (6:1) and then ethyl acetate-acetone (4:1) to give $\mathbf{1 0}$ (176 mg) and $\mathbf{1 1}(188 \mathrm{mg}$ ) in $21 \%$ and $15 \%$ yields, respectively. The HR-MS and NMR data of $\mathbf{1 0}$ and $\mathbf{1 1}$ are summarized in supporting information section.

\section{Preparation of $2^{\prime}, 5^{\prime}, 6^{\prime}-\operatorname{tris}[S-(N$-acetyl-O-methylcysteinyl $)]$ hydroxytyrosol}

(12) To a solution of di- $N$-acetyl- $O$-methylcysteine substituted hydroxytyrosol (11, 90 $\mathrm{mg})$ and $\mathrm{N}$-acetylcysteine methyl ester $(40 \mathrm{mg})$ in acetone $(50 \mathrm{~mL})$ was added a fine powder of DPPH $(140 \mathrm{mg})$ with stirring at $23{ }^{\circ} \mathrm{C}$. After stirring for several minutes, the solution was evaporated and subjected to column chromatography on silica gel (Cosmosil 75-SR-II-PREP, Nacalai) eluted with ethyl acetate-acetone (1:2) to give 12 $(60 \mathrm{mg}$ ) in 50\% yield. The HR-MS and NMR data of $\mathbf{1 2}$ are summarized in supporting information section.

\section{RESULTS AND DISCUSSION}




\section{Metmyoglobin-reducing activity of peroxide-catalyzed reaction products}

from polyphenols and cysteine. For the coupling of polyphenols with cysteine, we found that peroxidase-catalyzed oxidation gave the best result. ${ }^{9}$ Other methods that employ chemical reagents for this purpose either oxidized the cysteine first or required time-consuming follow-up to remove the reacted reagents. The MetMb-reducing activity of $40 \mu \mathrm{L}$ of the peroxidase reaction solutions, which were prepared from equimolar concentrations of polyphenol and cysteine, was measured using a freshly prepared MetMb solution $(60 \mu \mathrm{mol} / \mathrm{L})$. The data, which are summarized in Figure 1, show that most reaction products exhibited remarkable MetMb-reducing activity, whereas the peroxidase-oxidized polyphenols without cysteine did not show such high activity, except for the products from kaempferol and morin. Among the polyphenols examined, hydroxytyrosol, catechin, and caffeic acid showed the highest efficiency, followed by kaempferol, morin, taxifolin, rosmarinic acid, nordihydroguaiaretic acid. We had previously reported a high-MetMb reducing activity of polyphenols in the presence of cysteine ${ }^{5}$ suggesting that cysteinyl polyphenols produced in the solutions might play a role in MetMb-reducing activity. We therefore isolated cysteinyl polyphenols from the reaction mixtures and measured their ability to convert MetMb reductively to $\mathrm{MbO}_{2}$. 
Isolation and structure identification of cysteinyl polyphenols. From the

catechin reaction, two cysteinyl catechins (1 and 2) were detected using HPLC analysis and these were isolated using a preparative HPLC technique as described in the experimental section. High-resolution MS (HR-MS) of $\mathbf{1}$ and $\mathbf{2}$ revealed that they had the same molecular formula $\left(\mathrm{C}_{18} \mathrm{H}_{19} \mathrm{NO}_{3} \mathrm{~S}\right)$, indicating that both were isomers of mono-cysteine-substituted catechins. The substituted position was deduced by NMR data. The ${ }^{1} \mathrm{H}$ NMR data of $\mathbf{1}$ showed a coupling constant that signified ortho-related protons in the B-ring, indicating that the cysteine was substituted at the 2 "-position. C-H long-range correlations in HMBC of 1 were observed between $\mathrm{C}^{\prime}$ ' and $\mathrm{H} 2$, $\mathrm{C} 6{ }^{\prime}$ and $\mathrm{H} 2$, and $\mathrm{C} 2$ ' and $\mathrm{H} 2$ ", indicating that the substitution at position $2^{\prime}$ was through cysteinyl sulfur linkage. 1 was therefore determined to be 2 '-S-cysteinylcatechin (Figure 2). For the structure of 2, a meta-related coupling constant $(1.8 \mathrm{~Hz})$ was observed between $\mathrm{H} 2^{\prime}$ and $\mathrm{H}^{6}$ of the ${ }^{1} \mathrm{H}$ NMR data of B-ring. 2 was therefore determined to be 5'-S-cysteinylcatechin (Figure 2).

The peroxidase-catalyzed reaction of chlorogenic acid yielded a major coupling product (3), which had the molecular formula: $\mathrm{C}_{19} \mathrm{H}_{23} \mathrm{NO}_{11} \mathrm{~S}$ as derived from HR-MS data. The position of the substituted cysteine was determined from ${ }^{1} \mathrm{H}$ NMR data, which showed two ortho-coupling protons in a caffeate moiety. The structure of $\mathbf{3}$ was 
therefore determined to be 2 "-S-cysteinylchlorogenic acid (Figure 2).

Dihydrocaffeic acid gave major two coupling products (4 and $\mathbf{5})$ by the peroxidase-catalyzed reaction. These products were estimated to be di- and mono-cysteinyl dihydrocaffeic acid from the HR-MS data $\left(\mathrm{m} / \mathrm{z} 419.0572[\mathrm{M}-\mathrm{H}]^{-}\right.$and $300.0527[\mathrm{M}-\mathrm{H}]^{-}$, respectively). The substituted cysteine in mono-cysteinyl dihydrocaffeic acid (5) was estimated to occupy the 5'-position, because the meta-related coupling constant $(2.4 \mathrm{~Hz})$ was clearly observed in doublet signals of $\mathrm{H} 2^{\prime}$ and $\mathrm{H}^{\prime} .5$ was therefore determined to be $5^{\prime}$-S-cysteinyldihydrocaffeic acid. For the di-cysteine-substituted dihydrocaffeic acid (4), the ${ }^{1} \mathrm{H}$ NMR data were too complex to allow complete elucidation of the structure. An aromatic proton signal was observed at $7.01 \mathrm{ppm}$ as a singlet and NOE was observed from the aromatic proton to $\mathrm{H} 2$ and $\mathrm{H} 3$ of the cysteine part. These data indicated that the positions of the two substituted cysteines were either $2^{\prime}$ and $5^{\prime}$ or $5^{\prime}$ and $6^{\prime}$. Therefore, 4 was a di-cysteine-substituted dihydrocaffeic acid such as 2',5'-bis( $S$-cysteinyl)dihydrocaffeic acid or 5',6'-bis(S-cysteinyl)dihydrocaffeic acid (Figure 2) .

A peroxidase-catalyzed reaction of hydroxytyrosol in the presence of cysteine yielded two products (6 and 7). HR-ESIMS analysis of both products revealed that $\mathbf{6}$ and 7 were di-cysteine-substituted hydroxytyrosol and mono-cysteine-substituted 
hydroxytyrosol, respectively. The ${ }^{1}$ H-NMR spectrum of $\mathbf{6}$ was very complex except for the observation of a singlet aromatic proton at $7.03 \mathrm{ppm}$. We synthesized bis $[S$-(N-butoxycarbonylcysteinyl) $]$ hyroxytyrosol from hydroxytyrosol and $\mathrm{N}$-butoxycarbonylcysteine. In the compound, clear NOE between the aromatic proton and $\mathrm{H} 3$ of the cysteine part was observed, indicating that the substituted positions of the cysteines were either $2^{\prime}$ and $5^{\prime}$ or $5^{\prime}$ and $6^{\prime}$. Acid treatment of the di-( $N$-butoxycarbonyl)cysteinyl hydroxytyrosol gave $\mathbf{6}$. Therefore, 6 should be $2{ }^{\prime}, 5^{\prime}$ (or $\left.5^{\prime}, 6^{\prime}\right)$-bis(S-cysteinyl)hydroxytyrosol. Mono-cysteine substituted hydroxytyrosol (7) was also analyzed by NMR. Two meta-coupled aromatic protons were clearly observed in the ${ }^{1} \mathrm{H}$ NMR of 7 , revealing that 7 was $5^{\prime}$-S-cysteinylhydroxytyrosol (Figure 2).

Nordihydroguaiaretic acid, a potent antioxidant of creosote bush, also gave a coupling product $\mathbf{8}$ from the peroxidase reaction. The substituted position of the cysteine was determined to be at the 5'-position of one of the aromatic rings, which was revealed from the meta-related coupling constant between $\mathrm{H} 2{ }^{\prime}$ and $\mathrm{H} 6{ }^{\prime}$ in the ${ }^{1} \mathrm{H} \mathrm{NMR}$ of $\mathbf{8}$. Thus, $\mathbf{8}$ was determined to be 5'-S-cysteinylnordihydroguaiaretic acid (Figure 2). Rosmarinic acid, a labietae polyphenol, also yielded mono-cysteinyl rosmarinic acid (9) as a peroxidase-catalyzed reaction product. Rosmarinic acid has two tri-substituted benzene rings, however, the ${ }^{1} \mathrm{H}$ NMR data of 9 showed the existence of a 
four-substituted benzene ring possessing two protons with ortho coupling constant ( 8.4 $\mathrm{Hz}$ ). One of the protons of the benzene ring was relatively low-field shifted. Therefore, the substituted cysteine was determined to be at the 2-position of the benzene ring of a caffeoyl moiety of rosmarinic acid. Thus, 9 was 2 "'-S-cysteinylrosmarinic acid (Figure 2).

Metmyoglobin-reducing activity of cysteinyl polyphenols. Figure 3 shows the percent ratio of $\mathrm{MbO}_{2}$ produced in the reducing reaction of $60 \mu \mathrm{mol}$ of MetMb by $60-600 \mu \mathrm{mol}$ of cysteinyl polyphenols. All cysteinyl polyphenols examined showed remarkable and concentration-dependent reducing activity to MetMb for the first hour of the reaction. A decrease of $\mathrm{MbO}_{2}$ was observed in the reaction with cysteinyl polyphenols $\mathbf{5}, \mathbf{7}, \mathbf{8}$, and $\mathbf{9}$ after one hour. It should be noted that cysteinyl nordihydroguaiaretic acid (8) and cysteinyl rosmarinic acid (9) have two catechol structures and one of them remains in the intact form. We have reported that highly antioxidative polyphenols, most of which have the same catechol structure, enhance the oxidation of $\mathrm{MbO}_{2}$ in part owing to their pro-oxidant property. Therefore, the intact catechol structures of $\mathbf{8}$ and $\mathbf{9}$ should enhance the oxidative decrease of the initially produced $\mathrm{MbO}_{2}$. Cysteinyl polyphenols 5 (mono-cysteinyl dihydrocaffeic acid) and 7 (mono-cysteinyl hydroxytyrosol) have very similar structures. Although our previous 
results showed that a mono-sulfur substitution reduces the pro-oxidant properties of

highly reactive polyphenols ${ }^{4,5}$ it is possible that a mono-cysteine-substituted

dihyrocaffeic acid and hydroxytyrosol still have potent reactivity. ${ }^{10}$ These polyphenols

have a catechol structure with no conjugated electron-withdrawal groups, which have high reactivity and enhance both antioxidant and pro-oxidant activities. Therefore, the remaining pro-oxidative properties might exhibit oxidation of $\mathrm{MbO}_{2}$ at the end of the one-hour reaction. Noticeably, di-cysteine-substituted dihydrocaffeic acid (4) and hydroxytyrosol (6) did not show obvious pro-oxidant activity, which supports the idea that multi-sulfur substitution might reduce the pro-oxidant activity to $\mathrm{MbO}_{2}$, even in the presence of highly reactive polyphenols.

\section{The effect of the number of sulfur substitutions in polyphenols on}

metmyogolobin reduction and oxymyoglobin oxidation. Our previous results showed that in cysteinyl dihydrocaffeic acids and hydroxytyrosols, the number of sulfur substitutions have different effects and up to three substituents can be made to the benzene region. The question then arose as to how many cysteine substitutions would exhibit the best results in the terms of MetMb reduction and $\mathrm{MbO}_{2}$ production and retention. We attempted to synthesize tri-cysteine-substituted hydroxytyrosol, but were unsuccessful using the peroxide-catalyzed method as well as other chemical oxidation 
reagents. The reason for the failure seemed to be faster oxidation of the thiol part of the cysteine. We have reported that oxidation of the thiol part of the cysteine can be prevented by protection of both $\alpha$-amino and carboxylic groups. ${ }^{11}$ Using $\mathrm{N}$-acetylcysteine methyl ester $\left(\mathrm{NAC}-\mathrm{OCH}_{3}\right)$ as such a protectant, we prepared multi-sulfur-substituted hydroxytyrosols. The oxidative coupling of hydroxytyrosol with NAC-OCH $\mathrm{OCH}_{3}$ was performed in anhydrous acetonitrile using DPPH (2,2-diphenyl-1-picrylhydrazyl) as an oxidation reagent. The reaction of hydroxytyrosol with 4 equivalents of DPPH in the presence of equimolar $\mathrm{NAC}-\mathrm{OCH}_{3}$ yielded mono-substituted hydroxytyrosol (10) and di-substituted hyrdroxytyrosol (11) in 21\% and $15 \%$ yields, respectively. Additional DPPH oxidation of 11 with $\mathrm{NAC}-\mathrm{OCH}_{3}$ gave a tri-substituted hydroxytyrosol (12) in 50\% yield (Figure 4). The MetMb-reducing activities of 10, 11, and $\mathbf{1 2}$ were measured and the data are illustrated in Figure 5. The effects of NAC-substituted hyroxyltyrosols (10-12) and intact hydroxytyrosol on $\mathrm{MbO}_{2}$ are presented in Figure 6. Hydroxytyrosol and mono-cysteinyl derivative $\mathbf{1 0}$ showed no MetMb-reducing activity, whereas the di-cysteinyl derivative $\mathbf{1 1}$ and the tri-cysteinyl derivative 12 showed strong and concentration-dependent reducing activity. In particular, a higher reducing efficiency was observed in 11 (Figure 5), revealing that the di-sulfur-substituted compounds were the most effective at the reducing MetMb. In 
contrast, an additional substitution weakened the reducing activity of the di-substituted hydroxytyrosol. It should be noted that the reducing efficiency of the di-substituted hydroxytyrosol (11) reached almost a $100 \%$ for $3 \mathrm{~h}$ at $37{ }^{\circ} \mathrm{C}$ at a concentration of 600 $\mu \mathrm{mol} / \mathrm{L}$ versus $60 \mu \mathrm{mol} / \mathrm{L}$ of myoglobin. However, mono-substituted hydroxytyrosol (10)-induced enhancement of $\mathrm{MbO}_{2}$ oxidation was similar to that of hydroxytyrosol, while di- and tri-substituted hydroxytyrosols showed negligible enhancement (Figure 6). We have previously reported that the antioxidant capacity of a dihydrocaffeic ester was enhanced in the presence of a cysteine derivative. ${ }^{6}$ The antioxidation of dihydrocaffeic ester in the presence of a cysteine derivative in the production of mono-, di-, and tri-cysteinyl dihydrocaffeates. Furthermore, these mono- and di-substituted dihydrocaffeates mainly caused an increase in the length of the antioxidation period of dihydrocaffeate. Different reactivity to a peroxy radical was also observed among these cysteinyl dihydrocaffeates. These results clarified that the substitution of cysteine, and specifically a sulfur substitution, modulated the redox abilities of the original polyphenols. In the cysteinyl hydroxytyrosols, di-cysteine-substituted hydroxytyrosol (11) showed the most efficient reducing activity for MetMb and no prooxidant effect on $\mathrm{MbO}_{2}$. It is well known that hyroxytyrosol is an antioxidant present in olives and is also the antioxidant part of oleuropein ${ }^{12}$ which is the main polyphenol not only in young 
olive fruits ${ }^{13}$ but also in olive leaves. ${ }^{14}$ A series of investigations, including the current study, ${ }^{3-5}$ have demonstrated that potent active polyphenols could be used for preservation of fresh meat color in the presence of cysteine or related thiol compounds. We have also shown that a simple peroxidase-catalyzed reaction can be used for the modification of polyphenols to effectively reduce brown-colored MetMb. This simple method, which requires catalytic amount of enzyme and minimum amounts of cysteine and hydrogen peroxide, is applicable to advanced utilization of the raw extracts of polyphenol-rich plant resources such as olive leaves, because the reaction solution may be used directly for fresh meat color preservation.

Acknowledgments. This investigation was financially supported from The Ito Foundation (Tokyo, Japan) and JSPS Kakenhi (Grant Number 15K12326). 


\section{REFERENCES}

(1) Issanchu,S. Consumer expectations and perceptions of meat and meat product quality. Meat Sci. 1999, 43, S5-S19.

(2) Mancini, R. A.; Hunt, M. C. Current research in meat color. Meat Sci. 2005, 71, $100-121$.

(3) Masuda, T.; Inai, M.; Miura, Y.; Masuda, A.; Yamauchi, S. Effect of polyphenols on oxymyoglobin oxidation: Prooxidant activity of polyphenols in vitro and inhibition by amino acid. J. Agric. Food Chem. 2013, 61, 1097-1104.

(4) Inai, M.; Miura, Y.; Honda, S.; Masuda, A. Masuda, T. Metmyoglobin reduction by polyphenols and mechanism of the conversion of metmyoglobin to oxymyoglobin by quercetin. J. Agric. Food Chem. 2014, 62, 893-901.

(5) Miura Y.; Inai, M.; Honda, S.; Masuda, A.; Masuda, T. Reducing effects of polyphenols on metmyoglobin and the in vitro regeneration of bright meat color by polyphenols in the presence of cysteine. J. Agric. Food Chem. 2014, 62, $9472-9478$.

(6) Fujimoto, A.; Inai, M.; Masuda, T. Chemical evidence for the synergistic effect of a cysteinyl thiol on the antioxidant activity of caffeic and dihydrocaffeic esters. Food Chemistry, 2013, 138, 1483-1492. 
(7) Bowen, W. J. The absorption spectra and extinction coefficients of myoglobin, $J$.

Biol. Chem. 1949, 179, 235-245.

(8) Viriyarattanasak, C.; Hamada-Sato, N.; Watanabe, M.; Kajiwara, K. Suzuki, T.

Equation for spectrophotometric determination of relative concentrations of myoglobin derivatives in aqueous tune meat extracts. Food Chemistry 2011, 127, $656-661$.

(9) H. M. Awad, H. M.; M. G. Boersma, M. G.; Vervoorta, J.; Rietjens, I. M. C. M.

Peroxidase-catalyzed formation of quercetin quinone methide-glutathione adducts, Arch. Biochem. Biophys. 2000, 378, 224-233.

(10) Amorati, R.; Valgimigli, L.; Panzella, L.; Napolitano, A.; d'Ishia, M.

5-S-Lipoylhydroxytyrosol, a multidefense antioxidant featuring a solvent-tunable peroxy radical-scavenging 3-thio-1,2-dihydroxybenzene motif. J. Org. Chem.

2013, 78, 9857-9864.

(11) Miura, Y.; Honda, S.; Masuda, A.; Masuda, T. Antioxidant activities of cysteine derivatives against lipid oxidation in anhydrous media, Biosci. Biotechnol.

Biochem. 2014, 78, 1452-1455.

(12) Omar, S. H. Oleuropein in olive and its pharmacological effects. Sci. Pharm. 2010, $78,133-154$. 
(13) Amiot, M.-J.; Fleuriet, A.; Macheix, J.-J. Importance and evolution of phenolic compounds in olive during grows and maturation, J. Agric. Food Chem. 1986, 34, $823-826$.

(14) Savournin, C.; Baghdikian, B.; Elias, R.; Dargouth-Kesraoui, F.; Boukef, K.;

Balansard, G. Rapid high-performance liquid chromatography analysis for the quantitative determination of oleuropein in Olea eutropaea leaves. J. Agric. Food

Chem. 2001, 49, 618-621. 


\section{Figure Captions}

Figure 1. Effects of the products ( $600 \mu \mathrm{mol} / \mathrm{L}$ based on starting polyphenols) from polyphenols in peroxidase-catalyzed oxidations in the presence (red bar) or absence (blue bar) of cysteine on MetMb reduction. Results are expressed as mean \pm standard deviation (SD) (n=3). Compounds 1, 2'-S-cysteinylcatechin; 2, 5'-S-cysteinylcatechin; 3, 2"-S-cysteinylchlorogenic acid; 4, 2',5'-bis( $S$-cysteinyl)dihydrocaffeic acid; 5, 5'-S -cysteinyldihydrocaffeic acid; 6, 2',5' (or 5',6')-bis( $S$-cysteinyl)hydroxytyrosol; 7, 5'-S-cysteinylhydroxytyrosol; 8, 5'-S-cysteinylnordihydroguaiaretic acid; 9, 2"'-S-cysteinylrosmarinic acid.

Figure 2. Chemical structures of the cysteinyl polyphenols identified in peroxidase reaction solutions.

Figure 3. Effects of cysteinyl polyphenols $(\mathbf{1}-\mathbf{9})$ on MetMb reduction. All analytical experiments were performed in triplicate and the data were expressed as means (SDs were smaller than symbols).

Figure 4. Chemical structures of ( $N$-acetyl- $O$-methyl)cysteinyl hyrdoxytyrosols. 
Figure 5. Effects of (N-acetyl-O-methyl)cysteinyl hydroxytyrosols (10 - 12) and hydroxytyrosol on MetMb reduction. All analytical experiments were performed in triplicate and the data were expressed as mean \pm standard deviation (most SDs were smaller than symbols).

Figure 6. Effects of $(\mathrm{N}$-acetyl-O-methyl)cysteinyl hydroxytyrosols $(\mathbf{1 0}-\mathbf{1 2})$ and intact hyroxytyrosol as a reference compound with no-cysteine substitution on $\mathrm{MbO}_{2}$ maintenance. All analytical experiments were performed in triplicate and the data was expressed as mean \pm standard deviation (most SDs were smaller than symbols). 


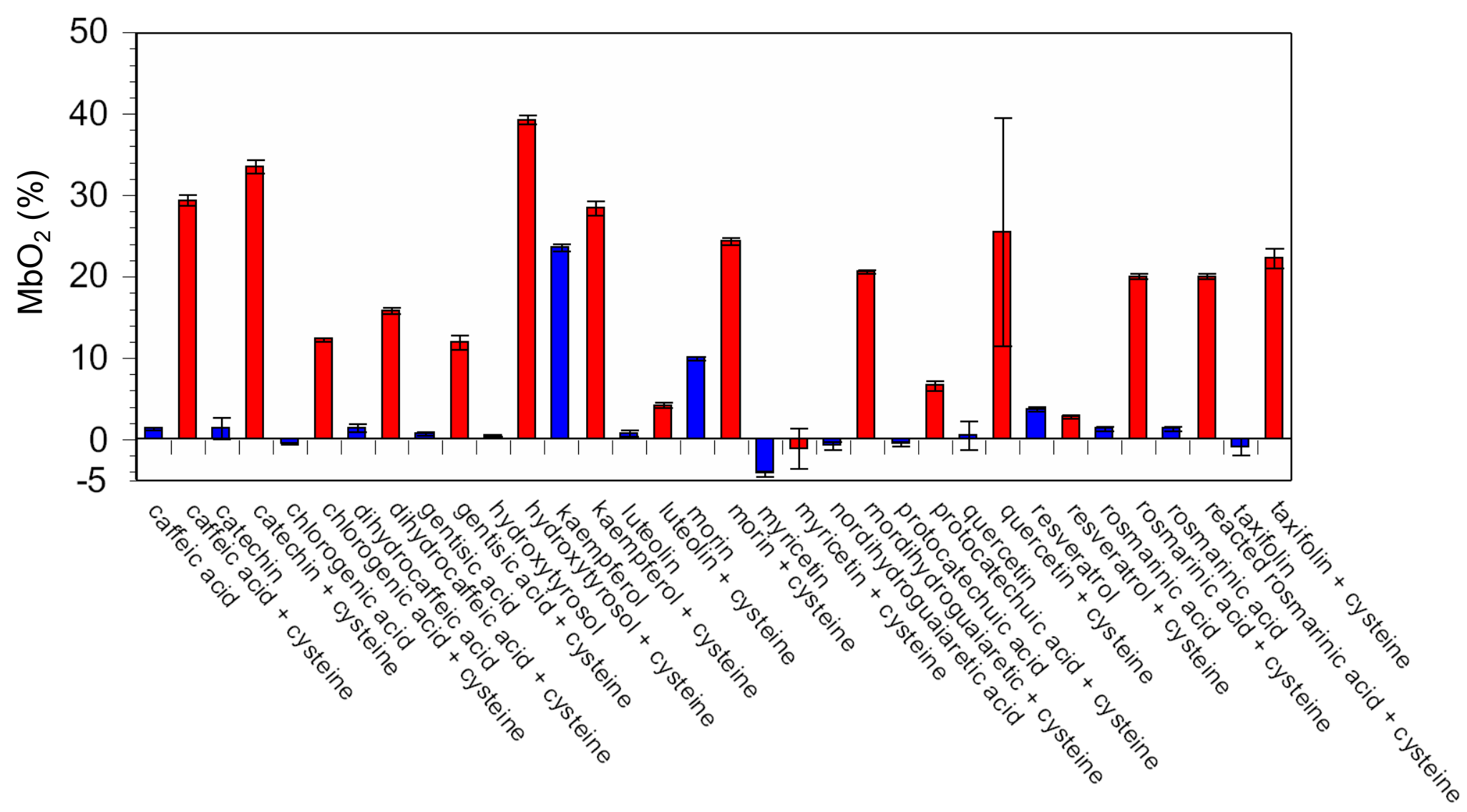




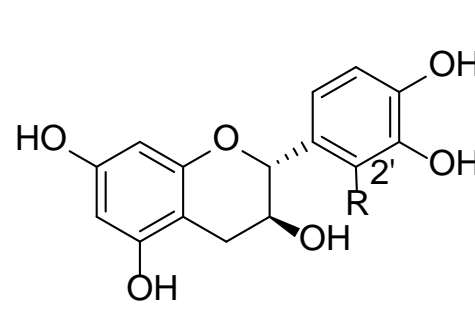

1<smiles>[R]c1cc(CCC(=O)O)c([R2])c(O)c1C</smiles>

or

4

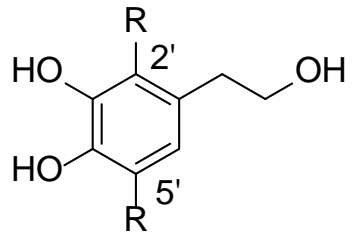

or

6<smiles>[R5]c1cc(CC(C)C(C)Cc2ccc(O)c(O)c2)cc(O)c1O</smiles>

8<smiles>[R5]c1cc([C@H]2Oc3cc(O)cc(O)c3C[C@H]2O)cc(O)c1O</smiles>

2<smiles>[R]c1c(CCC(=O)O)cc(O)c(O)c1[R]</smiles><smiles>[R]c1c(CCO)cc(O)c(O)c1[R]</smiles><smiles>[R2][Z20]([H])c1c(/C=C/C(=O)O[C@@H](Cc2ccc(O)c(O)c2)C(=O)O)ccc(O)c1O</smiles><smiles>[R2]c1c(/C=C/C(=O)O[C@H]2C[C@@]3(O)C[C@@H](O)[C@@H](O)[C@@H]2O3)ccc(O)c1O</smiles>

3

5<smiles>[R5]c1cc(CCO)cc(O)c1O</smiles>

Fig.2 

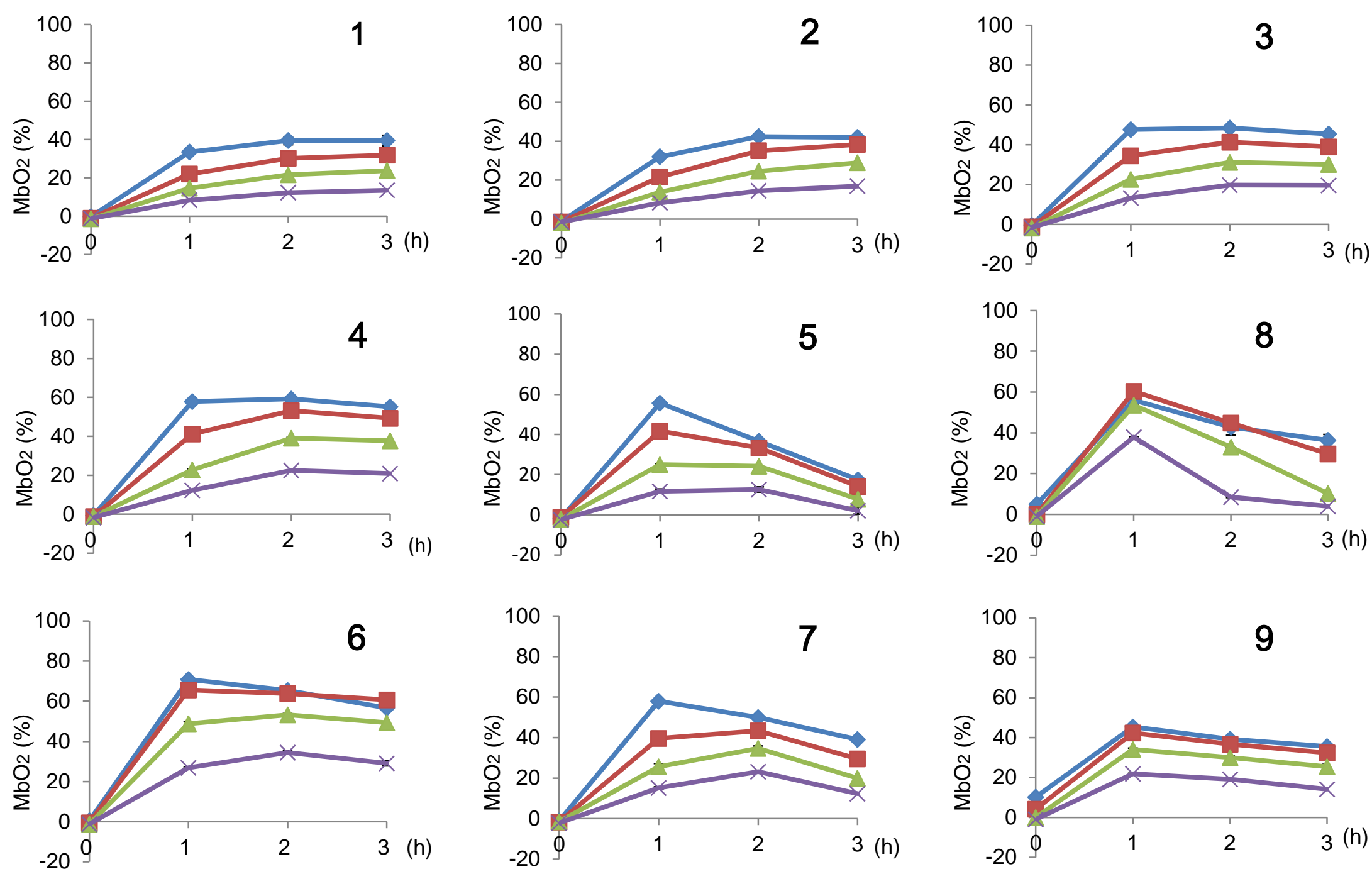

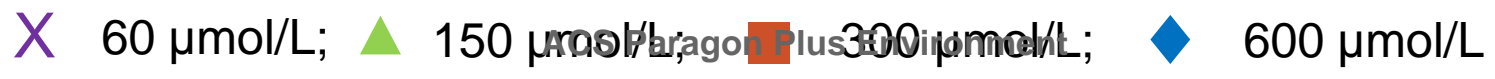

Fig.3 


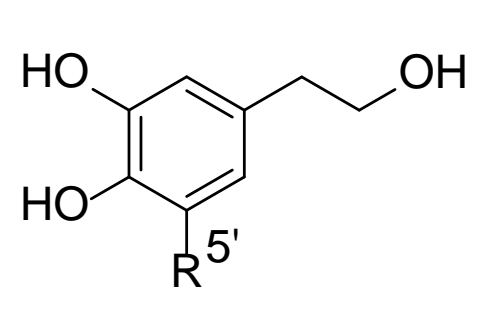

10

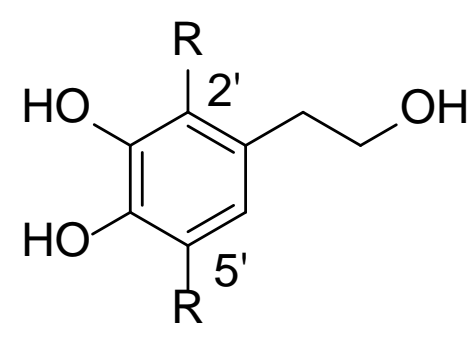

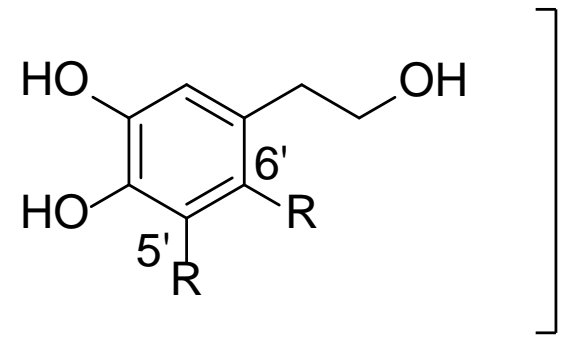

11
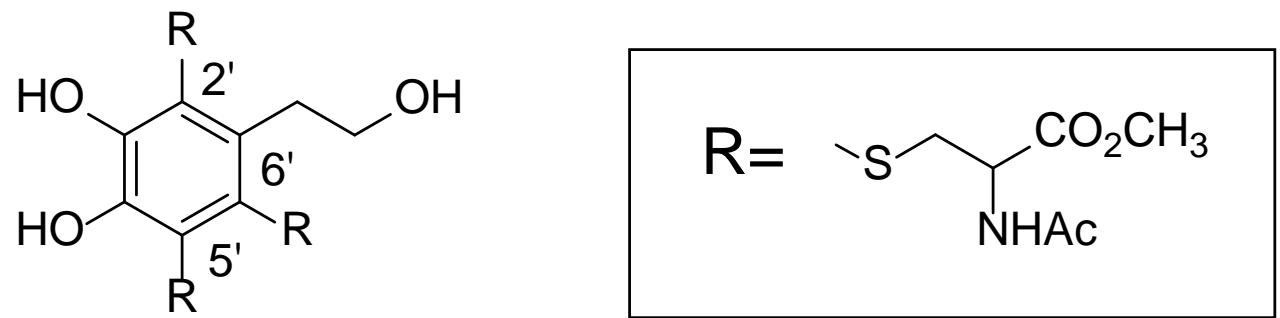

12

Fig. 4 

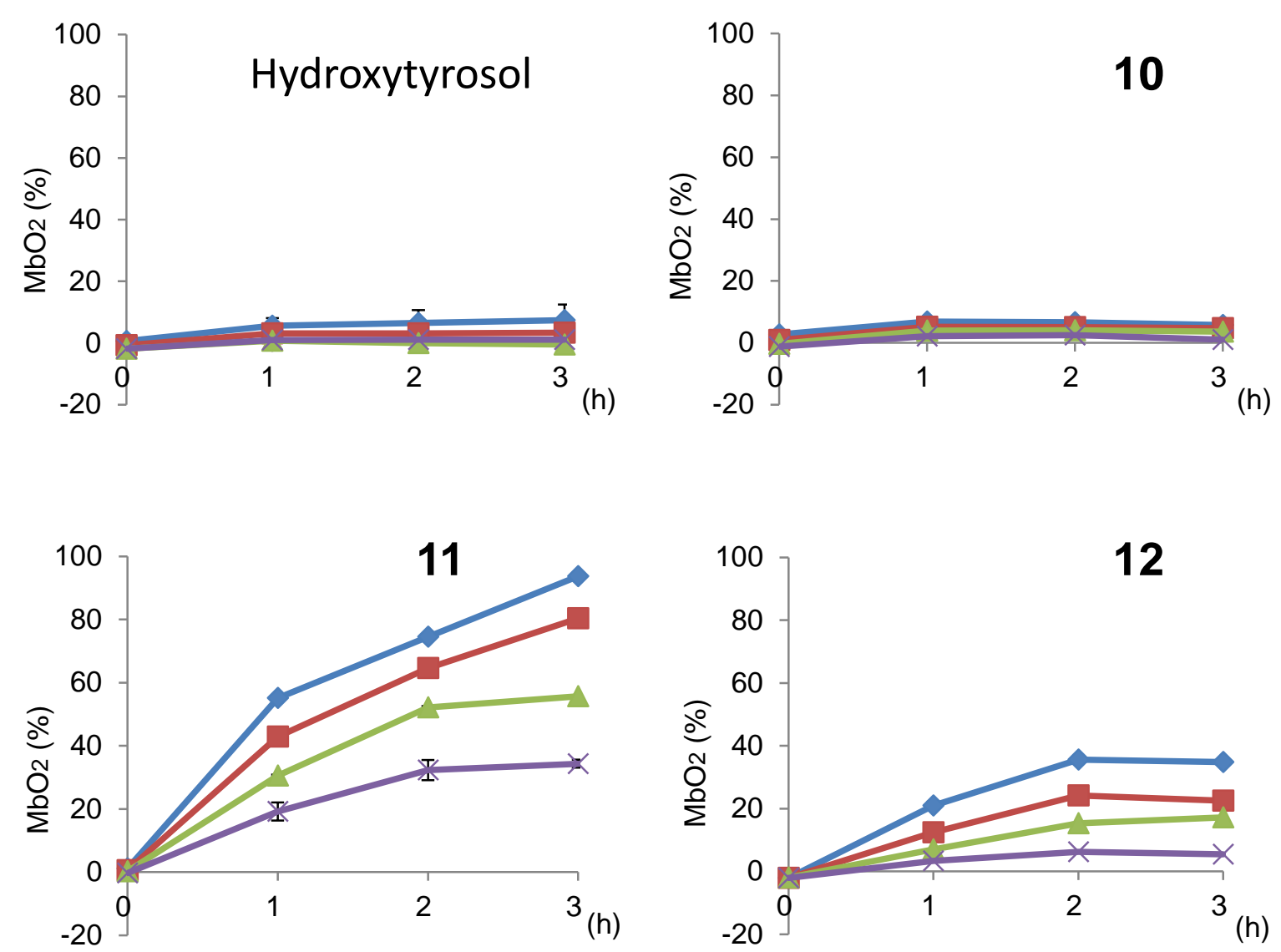

X $60 \mu \mathrm{mol} / \mathrm{L} ; \quad \triangle 150 \mu \mathrm{mol} / \mathrm{L} ; \quad \square 00 \mu \mathrm{mol} / \mathrm{L} ; \quad \diamond 600 \mu \mathrm{mol} / \mathrm{L}$ 

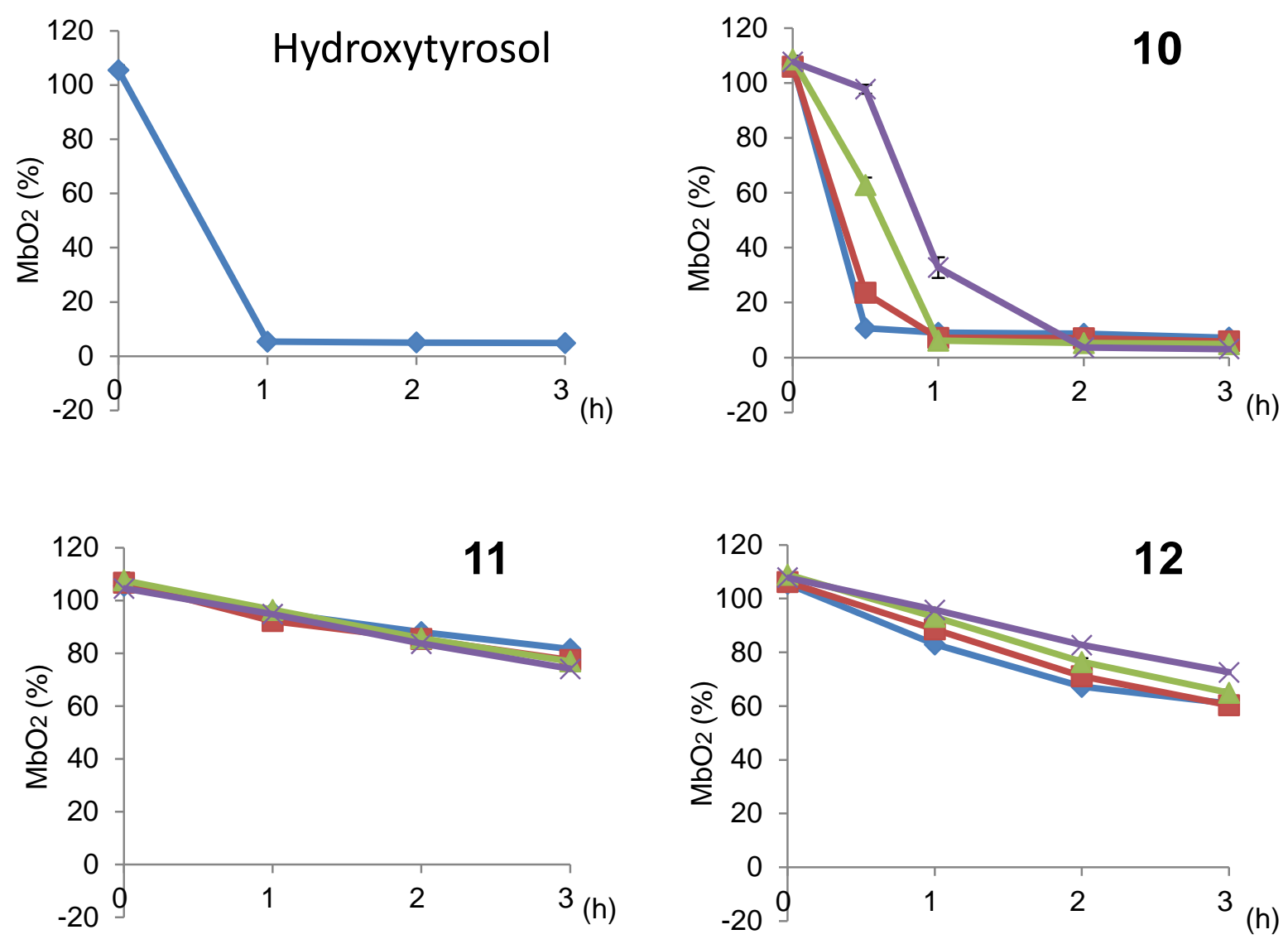

X $60 \mu \mathrm{mol} / \mathrm{L} ; \quad \triangle 150 \mu \mathrm{mol} / \mathrm{L} ; \quad \square 00 \mu \mathrm{mol} / \mathrm{L} ; \quad \diamond \quad 600 \mu \mathrm{mol} / \mathrm{L}$ 


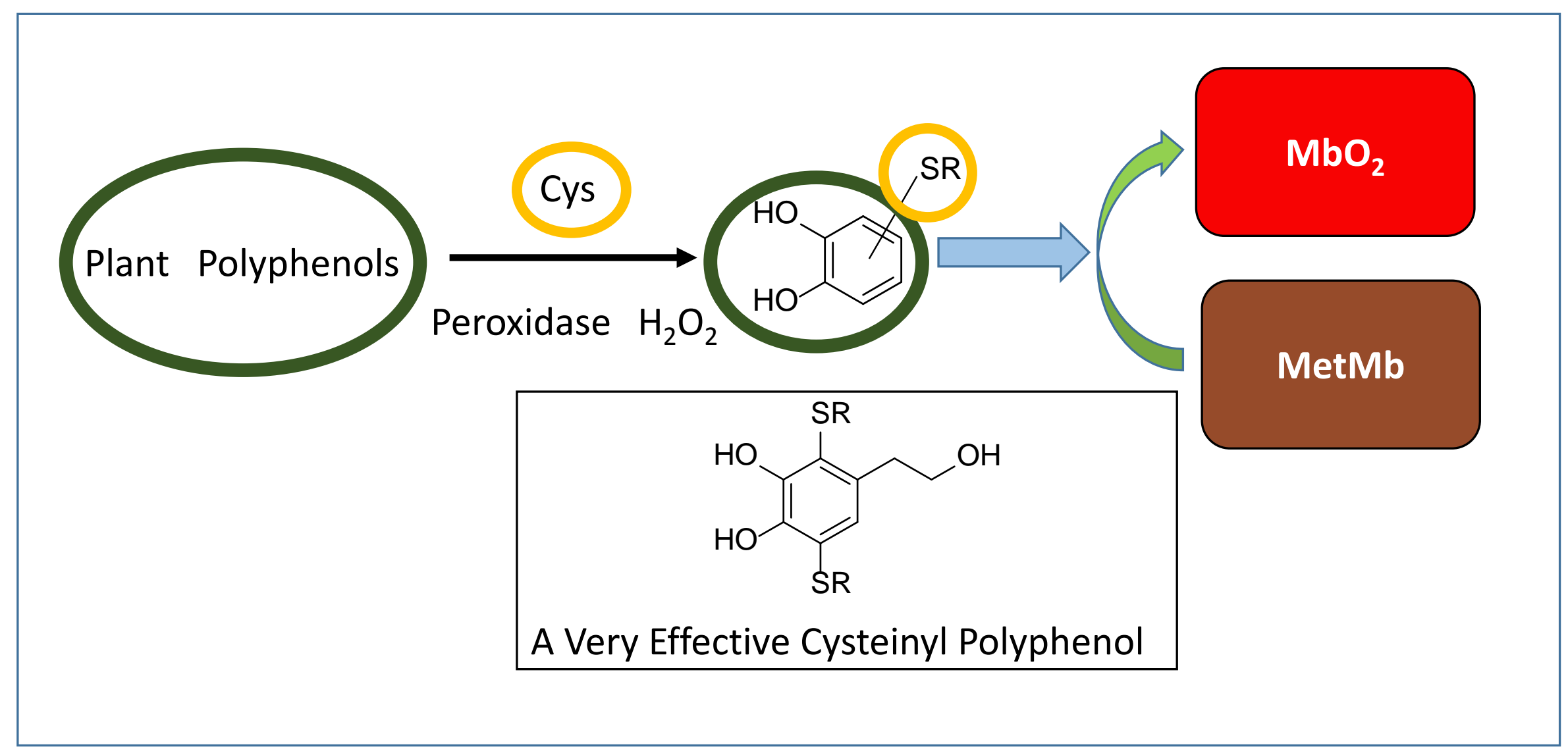

